\title{
Experimental and numerical characterization of the mechanical masseter muscle response during biting
}

\author{
J. Weickenmeier* \\ Department of Mechanical Engineering, Stanford University, Stanford 94305, USA \\ Department of Mechanical and Process Engineering, ETH Zurich, Zurich, Switzerland
}

M. Jabareen

Faculty of Civil and Environmental Engineering, Technion - Israel Institute of Technology, Haifa, Israel

B.J.D. Le Révérend

Nestlé Research Center, Rte du Jorat 57, CH-1000 Lausanne 26, Switzerland

\author{
M. Ramaioli \\ Department of Chemical and Process Engineering, University of Surrey, Guildford, UK \\ E. Mazza \\ Swiss Federal Laboratories for Materials Science and Technology - EMPA, Duebendorf, Switzerland \\ Department of Mechanical and Process Engineering, ETH Zurich, Zurich, Switzerland
}

Predictive simulations of the mastication system would significantly improve our understanding of temporomandibular joint disorders and the planning of cranio-maxillofacial surgery procedures. Respective computational models must be validated by experimental data from in-vivo characterization of the mastication system's mechanical response. The present pilot-study demonstrates the feasibility of a combined experimental and numerical procedure to validate a computer model of the masseter muscle. An experimental setup is proposed that provides a simultaneous bite force measurement and ultrasound-based visualization of muscle deformation. The direct comparison of the experimentally observed and numerically predicted muscle response demonstrates the predictive capabilities of such anatomically accurate biting models. Differences between molar and incisor biting are investigated; and muscle deformation is recorded for three different bite forces in order to capture the effect of increasing muscle fiber recruitment. The three-dimensional muscle deformation at each bite position and force-level is approximatively reconstructed from ultrasound measurements in five distinct cross-sectional areas (four horizontal and one vertical cross section). The experimental work is accompanied by numerical simulations to validate the predictive capabilities of a constitutive muscle model previously formulated. An anatomy-based, fully threedimensional model of the masseter muscle is created from

${ }^{*}$ Corresponding author: weickenmeier@stanford.edu magnetic resonance images of the same subject. The direct comparison of experimental and numerical results revealed good agreement for maximum bite forces and masseter deformations in both biting positions. The present work therefore presents a feasible in-vivo measurements system to validate numerically predicted masseter muscle contractions during mastication.

\section{Introduction}

Realistic and physics-based representations of the mastication system allow simulating biting, the impact of dental prosthesis, and surgical cranio-maxillofacial interventions. Reliable simulations require the experimental characterization of muscle properties and the quantification of interactions between soft tissues and bones. A quantitative estimate of intra- and inter-subject variability and anatomy-based geometric reconstructions of individual structures are important aspects in view of personalized medicine. Several such computational and experimental studies investigate temporomandibular joint disorders [2-4], surgical reconstruction of bone structures $[5,6]$, jaw movement during chewing [7, 8], and anatomical deficiencies causing dysphagia $[9,10]$.

The interplay of mastication muscles, as shown in Fig. 1, provides considerable jaw mobility and enables speech and mastication. Optimal nutritional uptake through efficient food processing begins with breaking down pieces and 
a)

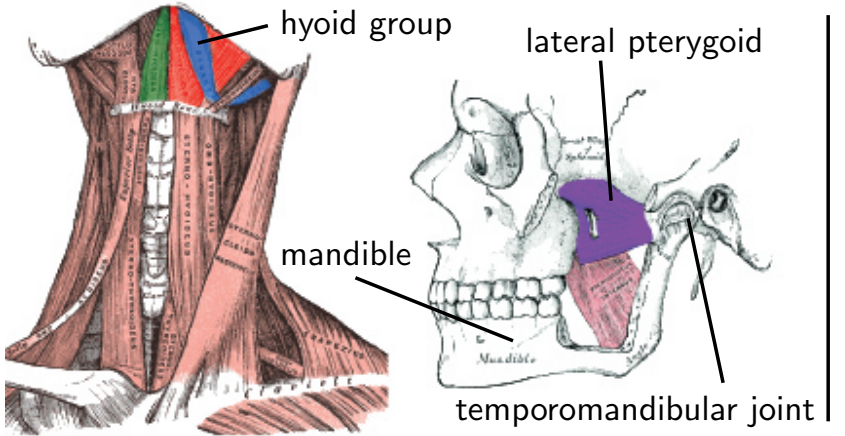

b)

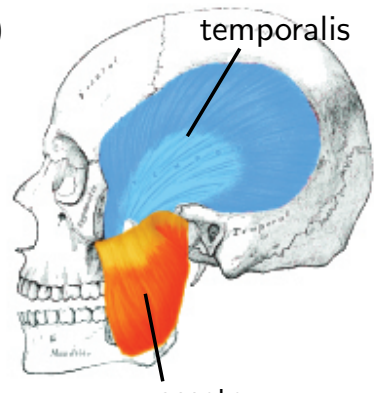

masseter

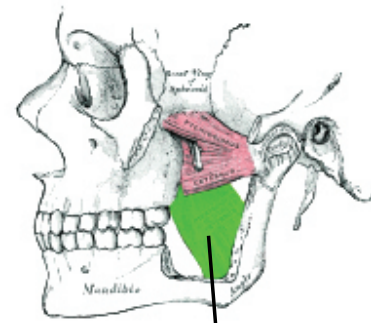

medial pterygoid

Fig. 1. Anatomical representation of the mastication system including the muscles of mastication, the hyoid muscle group, temporomandibular joint, mandible, and skull. The mastication muscles are often separated into the group of (a) jaw opening muscles consisting of the lateral pterygoid (purple) and hyoid muscle groups (red, blue, and green) and (b) jaw closing muscle group comprised of masseter (orange), temporalis (blue), and medial pterygoid muscle (green). Anatomical images adapted from Williams [1].

chewing in the mouth. This process includes different biting mechanisms that depend on food texture and bite size [11]. The human brain inherently controls bite force, biting position, and jaw motion through complex muscle activation patterns. Several mathematical models exist that investigate muscle activation patterns based on prescribed jaw kinematics [12-14], while others provide theoretical limits of bite forces for specific biting positions $[15,16]$. Most of these models, however, represent muscle forces by one-dimensional Hill-type springs [17], and therefore usually focus on determining singular points of muscle origin and insertion to establish a unique measure for muscle length [18]. The generalized approximation of three-dimensional tissue response, including not only forces but also tissue deformation, by one-dimensional material models limits the predictive capabilities of respective models for more complex biting cases [19]. Mastication models based on Hill-type springs alone cannot incorporate the geometry of the muscle and the contact interaction with surrounding bone and superficial tissue [20].

The experimental characterization of passive skeletal muscle $[21,22]$ as well as cartilage tissue of the temporomandibular joint disc [23-25] provides valuable insight into mechanical mastication muscle behavior. However, despite a number of theoretical and experimental studies on muscle deformation and muscle force [26,27], to this day, the mechanism of muscle shape changes is poorly understood. Muscle behavior is generally determined by fiber orientation and activation level. With respect to the human mastication system, only very few finite element (FE) models exists in literature, which provide a fully threedimensional representation including the mandible, skull, and masseter muscle [4,28]. Röhrle et al. [28] presented an anatomy-based reconstruction of the masseter muscle that is governed by transversely isotropic constitutive equations. The presented simulations predict muscle forces within a full biting cycle including jaw opening, closing, and biting. Commisso et al. [4] presented a detailed 3D reconstruction of the mastication system in order to study the stresses in the temporomandibular joint as well as the influence of the lateral pterygoid muscle on mandible movement during unilateral mastication. The model analyzed the sensitivity of activation patterns on the kinematics and loading of the jaw and temporomandibular joint. Böl et al. [27] introduced novel strategies for the evaluation of numerically predicted muscle deformations by comparison with MR images of the relaxed and contracted state and presented an experimental setup for real-time visualization of ex-vivo muscle contraction on a live animal model.

Preceding this work, Weickenmeier et al. [29] presented the numerical implementation of a constitutive muscle model proposed by Ehret et al. [30]. The mastication model used therein was derived from the anatomically detailed facial reconstruction developed by Barbarino et. al [31] and used to determine a physiological set of material parameters. Following this work, the present pilot-study investigates the feasibility of a procedure combining ultrasound and bite force measurements to validate numerical simulations of biting. It is shown that phenomenological constitutive equations and anatomically accurate masseter reconstructions are required to reliably predict bite forces and muscle deformations during clenching. In this regard, a combined experimental and numerical approach is proposed to investigate masseter muscle deformation during biting. An in-vivo setup for simultaneous bite force measurements and ultrasound-based visualization of muscle deformation is developed. In addition, a finite element model of the mastication system is generated based on medical images of the same subject from the experimental campaign. This allows for a direct comparison of the experimentally observed and numerically predicted mechanical masseter response. Validated and predictive computational mastication models can be utilized to study changes of forces in temporomandibular join disorders, to optimize maxillofacial surgery and predict the outcome of clinical procedures, and to support the development of novel food textures matched with the mastication abilities of specific population groups. 


\section{Materials and methods}

The analysis of masseter muscle mechanics includes numerical simulations and mechanical characterization during active contraction. The simulations are based on a finite element model of the masseter muscle. Mesh generation and material modeling are presented in Sections 2.1 and 2.2. The experimental setup for ultrasound image-based quantification of muscle deformation is described in Section 2.3 and an exemplary cross-sectional image is discussed in Section 2.4 .

\subsection{Finite element model generation}

The finite element model is created from magnetic resonance images of a 29 year old male. 180 transverse images are taken at a slice distance of $1 \mathrm{~mm}$ and an in-plane resolution of 256 pixels at a voxel size of $1 \mathrm{~mm}^{3}$. Scan IP software (Simpleware Ltd, Exeter, UK) is used to reconstruct the 3D contour of the masseter, mandible, and skull. Fig. 2a shows the initial reconstruction of the mastication structures before a semi-automatic meshing procedure is applied for finite element mesh generation. Figs. 2d-h illustrate the stepwise transformation of the initial medical image segmentation into a homogeneous and refined mesh. Starting with the triangulated surface of the outer masseter contour (d), Geomagic Studio software (3D Systems, Rock Hill, SC, US) is used to generate a patched surface represented by nonuniform rational B-splines (e). Given the flat shape and regular thickness of the masseter muscle, the outer surfaces are approximated by two opposing faces which are patched by matching grids (f). Using a user subroutine, corresponding quadrilateral shells of front and back faces are combined to form hexahedral elements (g). This strategy allows for multiple uniform mesh refinement steps while preserving a favorable aspect ratio. The masseter model, which is used for all simulations in the present work, consists of 6144 linear 8-noded hexahedral elements (h). The commonly accepted assumption of a nearly incompressible material behavior requires reduced integration with hourglass stabilization. The corresponding reduced integration elements (C3D8R) prevent volumetric locking while reducing computational cost, due to the evaluation of the constitutive equations at a single integration point per element [29]. The mesh is highly homogenous and was refined to ensure convergence of results with respect to spatial discretization. Skull and mandible are reconstructed from the medical images, smoothed, and converted into non-uniform rational basis splines (NURBS) surfaces to serve as display bodies in the model (b). Finally, the muscle mesh and bone surfaces are imported into the commercial finite element program Abaqus [32] (Dassault Systémes, Providence, RI, USA) for numerical simulations of the mastication model (b).

For computational efficiency and the absence of representative boundary conditions for asymmetric biting, only half of the head is modeled. This symmetry assumption must be considered when comparing numerical simulations to experiments of unilateral biting. In fact, several studies look into the effect of unilateral biting on bite forces and loads on the ipsilateral and contralateral temporomandibular joints [33-35]. In the present model, masseter and skull are constrained on the basis of anatomical findings illustrated in the textbook by Williams [1]. Muscles of mastication have one rigid insertion point and one insertion point with multiple degrees of freedom. As shown in Fig. 2c, the nodes on the cranial end of the masseter muscle are fixed, while the caudal nodes are tied to the degrees of freedom of the condyle head, see Fig. 2c. Non-penetrating constraints between masseter muscle and bones are enforced to exclude intersections upon muscle contraction. Contact of incisor and molar teeth is detected by interaction constraints between sensor elements placed in the teeth of the upper and lower jaw that come into contact upon biting. These elements have stiff elastic properties (elastic modulus: 1GPa, Poisson's ratio: 0.499) which allow to extract the bite force vector based on the contact forces. Rough contact properties are assigned to these sensor elements such to ensure no relative movement between teeth once they have come into contact.

Biting is modeled as a quasi-static, two-step simulation of closing the mouth followed by clenching. As described in more detail in Section 3.2, the first step includes pure translation of the mandible to align the teeth that will be in contact, i.e. molar versus incisor biting, followed by a pure rotation of the mandible about the condyle head upon muscle contraction, as shown in Figs. 2c and 5. A total simulation time of $0.3 \mathrm{~s}$ is required with $0.1 \mathrm{~s}$ for jaw alignment and $0.2 \mathrm{~s}$ to reach maximum bite force. Adaptive time stepping with a maximum time increment of $0.0001 \mathrm{~s}$ is used and ensures convergence for the entire muscular contraction step.

\subsection{Material modeling}

Voluntary contraction in skeletal muscles is controlled by the somatic nervous system while the overall mechanical muscle response depends on its current kinematical configuration and the level of activation in terms of accumulated impulses sent to the muscle. Based on a physically motivated three-dimensional constitutive model for skeletal muscle proposed by Ehret et al. [30], the strain energy function $W$ considered here takes the following form

$$
\begin{aligned}
& W=\frac{1}{4} \mu\left\{\frac{1}{\alpha}(\exp [\alpha(\tilde{I}-1)]-1)\right. \\
& \left.+\frac{1}{\beta}(\exp [\beta(\tilde{J}-1)]-1)+\frac{1}{\gamma}\left(I I I_{C}^{-\gamma}-1\right)\right\}, \\
& \tilde{I}=\mathbf{C}:\left(\tilde{\mathbf{L}}+w_{a} \mathbf{M}\right), \tilde{J}=\operatorname{cof}(\mathbf{C}): \tilde{\mathbf{L}}, I I I_{C}=\operatorname{det}(\mathbf{C}),
\end{aligned}
$$

where $\mu, \alpha, \beta, \gamma$ are material parameters [29]. $\tilde{I}, \tilde{J}$, and $I I I_{C}$ are three invariants of the right Cauchy Green tensor $\mathbf{C}=$ $\mathbf{F}^{T} \mathbf{F}$, and the generalized tensor $\tilde{\mathbf{L}}$ is given by

$$
\tilde{\mathbf{L}}=\frac{w_{0}}{3} \mathbf{I}+w_{p} \mathbf{M} .
$$

The structural tensor $\mathbf{M}$ governs the anisotropic material behavior and takes the form $\mathbf{M}=\mathbf{m} \otimes \mathbf{m}$, where $\mathbf{m}$ is the unit 
a)

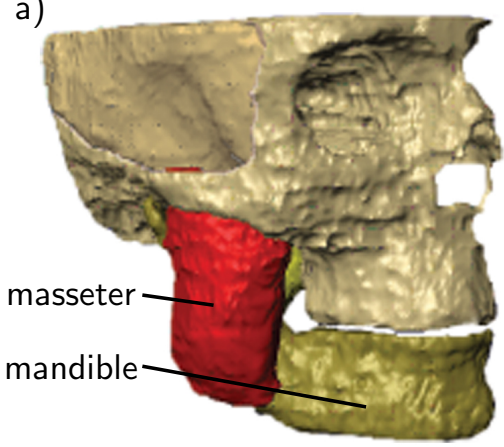

d)

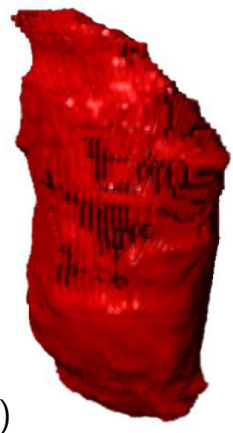

e)

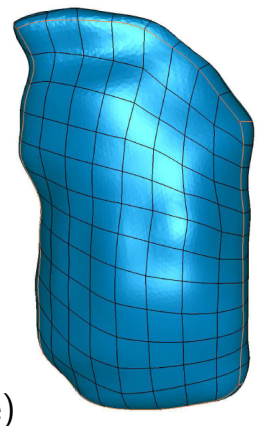

b)
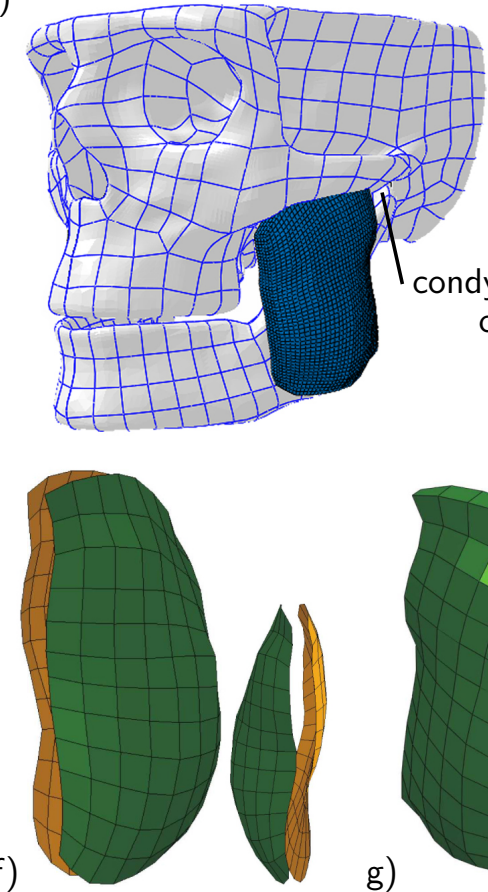

c)

fixed boundary condition

g)

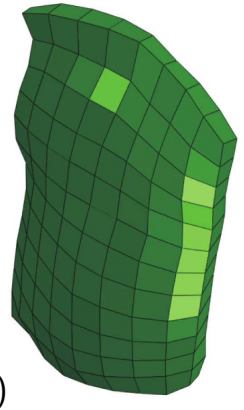

h)

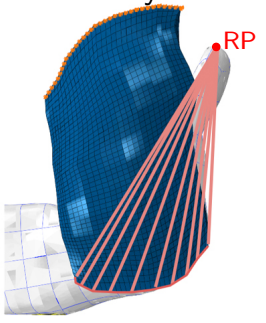

kinematic coupling of masseter to the condyle head

Fig. 2. Finite element model generation of the mastication system. a) Magnetic resonance image-based segmentation of skull, mandible, and the masseter muscle, b) anatomically detailed representation of bone structures and the masseter, c) boundary conditions defining the kinematic constraints on the masseter muscle: nodes at the top of the masseter are fixed to represent the anchoring with the cranium; bottom nodes are kinematically coupled to the degrees of freedom of the condyle head of the temporomandibular joint (TMJ). In the finite element model a reference point (RP) is defined on the condyle head which controls the degrees of freedom of the rigid mandible. Images d) through h) visualize individual steps in the semi-automatic procedure of mesh development. The rough outer contour of the muscle is transformed into a sufficiently refined, hexahedral finite element mesh. The finest mesh with 6144 elements $(\mathrm{h})$ is used for all subsequent simulations.

vector parallel to the preferred muscle fiber direction in the reference configuration. The identity tensor $\mathbf{I}$ describes the passive isotropic response of the muscle tissue, while the weighing parameters $w_{0}$ and $w_{p}$ govern the ratio between isotropic muscle matrix material and muscle fibers, respectively, with $w_{0}+w_{p}=1$. The active muscle contribution is introduced through parameter $w_{a}$, which affects the generalized invariant $\tilde{I}$, is based on the work presented by Corless et al. [36], and takes the form

$$
w_{a}=\left\{\begin{array}{ll}
0 & \text { if } P_{a c t}=0, \\
\frac{W_{0}\left(\chi^{\star}\right)}{\alpha \lambda_{m}^{2}}-\frac{\tilde{I}_{p}^{\prime}}{2 \lambda_{m}} & \text { else, }
\end{array},\right.
$$

where $W_{0}\left(\chi^{\star}\right)$ denotes the solution for the principal branch of the Lambert- $W$ function and $\chi^{\star}$ is given by

$$
\begin{aligned}
\chi^{\star}= & P_{a c t} \frac{2 \alpha \lambda_{m}}{\mu} \exp \left[\left(\frac{\alpha}{2}\right)\left(2-2 \tilde{I}_{p}+\lambda_{m} \tilde{I}_{p}\right)\right] \\
& +\frac{\alpha}{2} \lambda_{m} \tilde{I}_{p} \exp \left[\left(\frac{\alpha}{2}\right) \lambda_{m} \tilde{I}_{p}\right] .
\end{aligned}
$$

The argument of the Lambert- $W$ function $\chi^{*}$ depends on the squared fiber stretch $\lambda_{m}=\sqrt{\mathbf{C}: \mathbf{M}}$, the nominal stress $P_{a c t}$ due to the muscle activation, and the first derivative of the purely passive part of the first invariant $\tilde{I}$ with respect to fiber stretch for the case of uniaxial stretch. Specifically, $\tilde{I}_{p}^{\prime}$ takes the form

$$
\tilde{I}_{p}=\frac{\partial \tilde{I}_{p}}{\partial \lambda_{m}}=\frac{w_{0}}{3}\left(2 \lambda_{m}-\frac{2}{\lambda_{m}^{2}}\right)+2 w_{p} \lambda_{m}
$$

The nominal stress due to muscle activation [30] is proposed in the following form

$$
P_{a c t}=f_{\zeta} \cdot f_{\mathrm{v}} \cdot N a \sum_{i=1}^{n_{\mathrm{MU}}} \rho_{i} F_{t}^{i},
$$

and depends on the level of activation, sarcomere length (function $f_{\zeta}$ ), and contraction velocity (function $f_{v}$ ), the measure of the total number of activated motor units per reference cross-sectional area $N_{a}$, the total motor unit types $n_{\mathrm{MU}}$, the corresponding fraction of each motor unit $\rho_{i}$, and the twitch force of a single motor unit $F_{t}^{i}$. Weickenmeier et al. [29] present the comprehensive numerical implementation of this material model in order to enable the realistic simulation of geometrically complex muscle structures in the face and forehead region [37]. In the present work, material parameters proposed by Weickenmeier et al. [29] in 


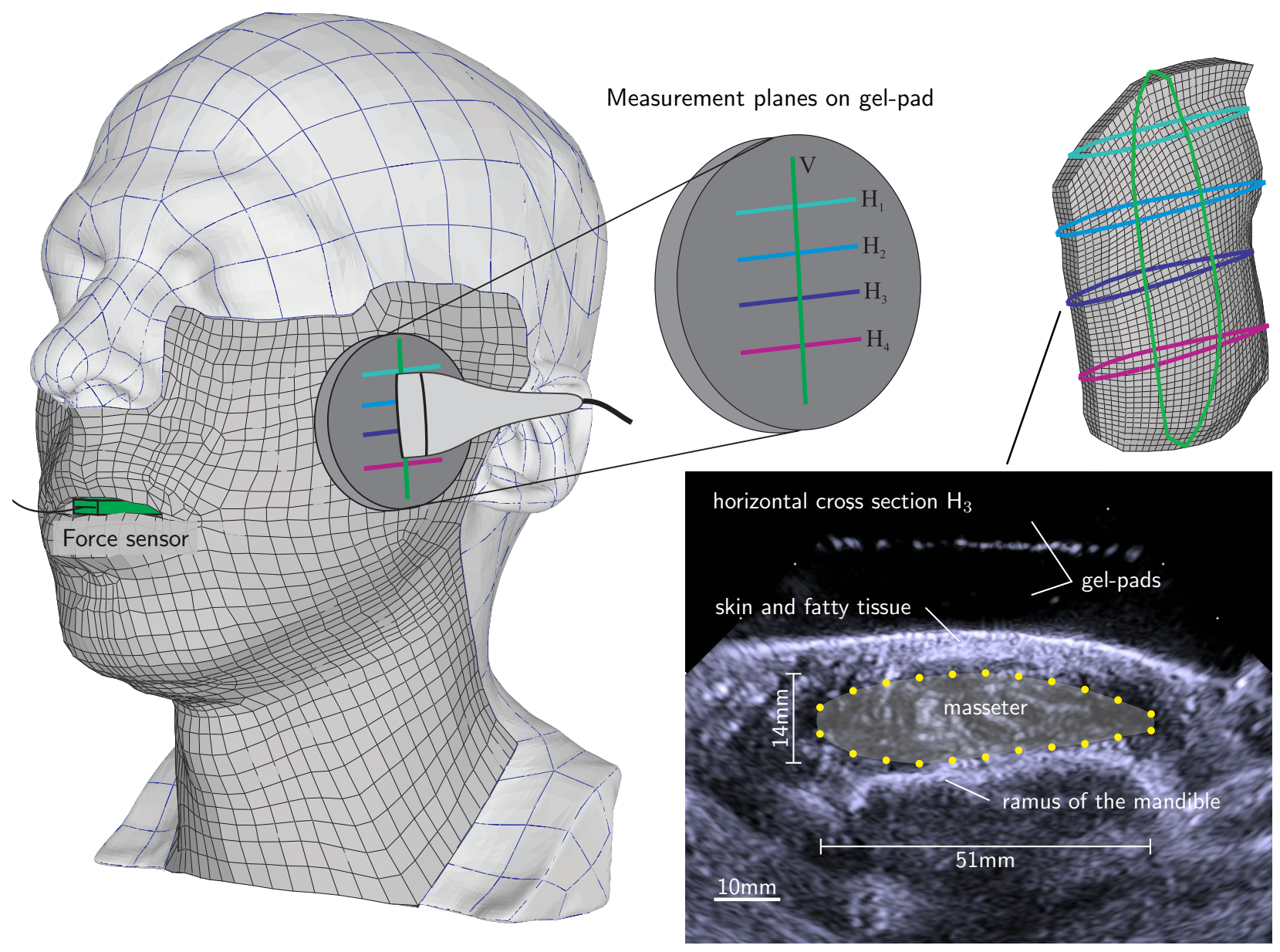

Fig. 3. Illustration of the experimental setup for the measurements of muscle deformation during contraction. The force sensor allows for real-time measurements of the current bite force in the specific biting locations. At the same time, ultrasound imaging is used to capture muscle shape changes in multiple cross sections of the muscle depending on biting position and bite force. Three dimensional muscle shape is approximated by six measurement planes as indicated on the gel pad and finite element mesh of the masseter. The ultrasound image shows the relaxed horizontal cross-section of the masseter muscle in position $\mathrm{H}_{3}$. Yellow points indicate the outer contour of the masseter muscle. Muscle dimensions are measured for comparison with values reported in literature. Standoff gel-pads, skin and fatty tissue layers are clearly visible and the ramus of the mandible appears as a white layer inferior to the muscle's cross section.

Tables I and II are used in all simulations.

\subsection{Experimental setup}

An experimental setup for synchronized bite force measurements and the visualization of masseter deformation in particular cross sections of the muscle was developed. Ultrasound imaging is used to capture cross-sectional shape changes of the muscle at various locations and at different bite forces. Moreover, in order to differentiate between two very different biting cases, a pressure sensor is placed either between the frontal incisors or on a specific side between the molars respectively. Both cases have noticeable differences with respect to muscle involvement, maximum bite force, and muscle deformation during force generation. Bite forces are measured with a flexible force sensor (Flexiforce A201, Tekscan, Boston MA, USA) up to a maximum acceptable load of $445 \mathrm{~N}$. The $15 \mathrm{~mm}$ x $15 \mathrm{~mm}$ sized Flexiforce sensor is placed between two $15 \mathrm{~mm} \times 15 \mathrm{~mm} \times 0.4 \mathrm{~mm}$ sized rigid stainless steel plates and then embedded in a $30 \mathrm{~mm} \mathrm{x}$ $10 \mathrm{~mm} \times 5 \mathrm{~mm}$ block of dental grade silicon (Aquasil Ultra $\mathrm{M}+\mathrm{W}$ Dental Swiss AG, Illnau, $\mathrm{CH}$ ) in order to evenly distribute the bite force exerted on the sensor by the heterogeneous occlusal surface of the teeth. The gap (10 $\mathrm{mm}$ at rest) resulting from biting on the silicon block mimics the chewing process of food at a physiological jaw opening angle. A Noraxon Myosystem 1400L (Velamed, Germany) fitted with a Noraxon in-line adapter model 324 (Velamed, Germany) was used as an analog/digital converter to record the force measured by the FlexiForce sensor. The linearity of the sensors was verified prior to the experiments by applying an external calibration procedure using a TAXT+ (Stable Microsystems, UK) equipped with the geometry SMSP/20 with known loads between 10 and $450 \mathrm{~N}$. All sensors responded linearly $\left(r^{2}>0.98\right)$.

Fig. 3 shows the experimental setup for measurements of 
bite force and transient muscle deformations during contraction. The three-dimensional shape change of the muscle is approximated by the muscle's cross-sectional area changes in four evenly spaced transverse planes $\left(H_{1}-H_{4}\right)$ and one vertical plane $(V)$ in the middle of the muscle (Fig. 3). An $8 \mathrm{MHz}$ linear array ultrasound probe (Siemens ACUSON 8V3 probe) is used to take B-mode image sequences with an in-plane resolution of $0.1 \mathrm{~mm} /$ pixel (254dpi). 30s video sequences at 50 frames/s capture the muscle deformation during multiple biting cycles. Each cycle includes the buildup and relaxation of a specific bite force. Four distinct features of the setup provided reproducible ultrasound measurements: Markers on the gel-pad ensured repeatable probe placement; during measurements, the subject's head was tilted sideways to prevent shifting of the gel-pad and to minimize probe movement by the operator; a thick layer of ultrasound gel between pad and probe and the self-weight of the probe minimized the necessary contact pressure throughout the biting cycle, and in the relaxed state in particular; and the gap between cheek and probe allowed the operator to maintain probe orientation throughout the 30s video without interfering with muscle contraction. Reliable reconstruction of muscle deformation from ultrasound videos was most sensitive to probe motion during biting and only marginally affected by changes in the (low) probe contact pressure. The relation between bite force and muscle deformation was determined for three physiological bite force magnitudes: $50 \mathrm{~N}, 100 \mathrm{~N}$, and $200 \mathrm{~N}$. During each video sequence, the subject is required to reach and briefly maintain the prescribed bite force in at least two biting cycles.

In total, 75 video sequences were recorded to test the masseter response for two biting positions (molar and incisor), for three bite forces $(50 \mathrm{~N}, 100 \mathrm{~N}$, and $200 \mathrm{~N})$, and for five cross-sectional muscle planes $\left(V\right.$ and $\left.H_{1}-H_{4}\right)$. Repeatable probehead placement for the five different measurement planes is ensured through markers on the ultrasound standoff gel-pad placed on the subject's cheek, as shown in Fig. 3. Muscle deformation, and cross-sectional area changes specifically, are extracted from the ultrasound image sequences using an optical flow tracking algorithm previously presented by Weickenmeier et al. [37]. After a manual segmentation of the muscle contour in the first frame of the image sequence, the optical flow algorithm tracks all points in each consecutive frame. For every frame, the crosssectional area is defined by the convex hull around all tracked points.

\subsection{Ultrasound imaging of masseter muscle}

The resolution of the ultrasound image allows to differentiate between a highly echogenic epidermal layer $(\sim 1 \mathrm{~mm})$ and a thicker layer of dermis and subepidermal tissue ( $\sim 5 \mathrm{~mm})$ characterized by a lower echogenicity. As shown through the example of the horizontal plane $\mathrm{H}_{3}$ in Fig. 3, the masseter is clearly visible and surrounded by a thin fascial membrane that appears as a strongly echogenic band around the muscle [38]. Based on other studies in literature, it is expected that the muscle-tendon complex at the cranial end of the masseter muscle is not fully differentiable in the US image given limitations by the field of view and disturbances of the ultrasound signal by the neighboring zygomatic arch. As a result the vertical muscle cross-sectional area is underestimated in the experimental study while much better represented in the finite element model obtained from MR images, see Fig. 2b and Fig. 6. In particular, Kubo et al. [34] reported generally larger cross-sectional area and thickness measurements based on MR images in comparison to ultrasound images. Raadsheer et al. [39] suggest that a common source for larger MRI based values is related to an underrepresented aponeurotic tissue layer surrounding the muscle in ultrasound images.

\section{Results}

\subsection{Muscle deformation during biting}

Fig. $4 \mathrm{a} / \mathrm{b}$ shows the cross-sectional area change during biting observed in the horizontal plane $\mathrm{H}_{3}$ for a) molar and b) incisor biting and the three bite forces. The proposed contour tracking algorithm provides a reliable measure for area change with respect to the reference cross-sectional area as well as the area change during biting, as shown by the experimental results for all six respective measurements. $K$ means clustering is used to separate the cyclic area data into two subsets, as shown through the example of one curve in the inlet of Fig. 4a by the orange and gray measurement points. The median of each set of measurement points, indicated by the orange and gray dashed lines, provides the cross-sectional area in the relaxed state in-between biting cycles and the area at the prescribed bite force, respectively.

Fig. 4c/d shows the experimentally realized median area values for each bite force level in the five different cross sections. Error bars indicate the median absolute difference and quantify the variability in the area calculation. Variability is primarily determined by the subject's ability to maintain the prescribed bite force throughout multiple cycles. Involuntary minor muscle contractions to ensure proper sensor positioning in-between individual biting sequences result in median absolute difference values of up to three times larger in comparison to the better defined biting state, see Fig. $4 \mathrm{a} / \mathrm{b}$.

It is generally observed that all horizontal cross sections increase with bite force although the magnitude of area change is clearly location dependent. The muscle's largest horizontal cross section of $698 \mathrm{~mm}^{2}$ is found in the lower half $\left(\mathrm{H}_{3}\right)$ and thins, i.e. decreases in area, towards its distal ends by $47 \%$. Especially medial horizontal cross sections increase most $\left(\mathrm{H}_{3}: 8 \%\right.$ at $50 \mathrm{~N}, 11 \%$ at $100 \mathrm{~N}$, and $12 \%$ at $200 \mathrm{~N}$ with respect to the reference area) which is related to the fact that the local anatomy is characterized by a significantly denser muscle fiber network in comparison to the distal ends of the masseter. And finally, in the case of incisor biting the muscle's area, and primarily its lateral thickness, increases much stronger for the same bite force in comparison to the molar case: $15.5 \%$ for the incisor case in comparison to $8.5 \%$ during molar biting. These findings need to be interpreted in view of the mastication system's kinematics and are discussed in Section 4. 
(a) Change of area $\mathrm{H}_{3}$ during molar biting

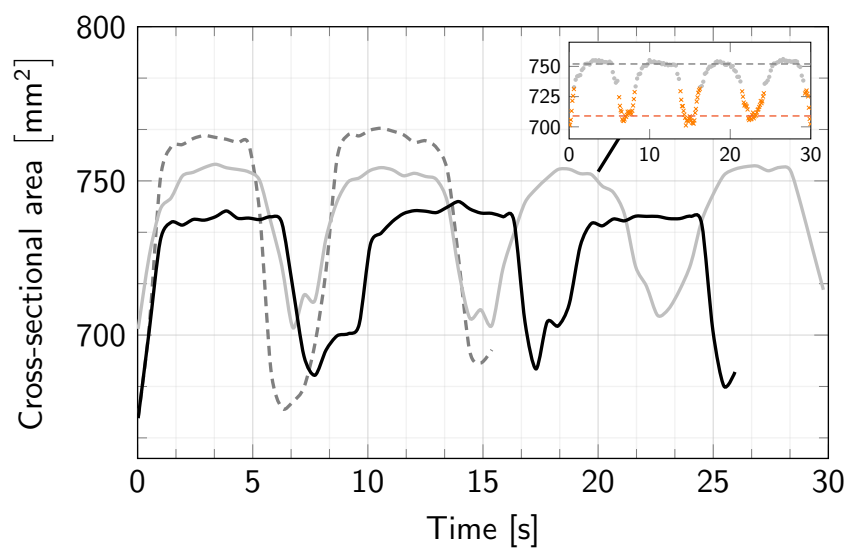

(b) Change of area $\mathrm{H}_{3}$ during incisor biting

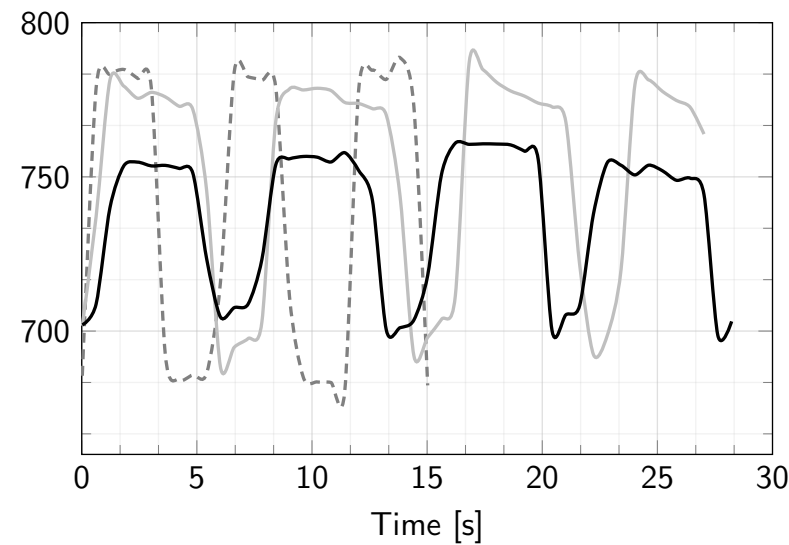

- Bite force $50 \mathrm{~N}$ — Bite force $100 \mathrm{~N}$ - - - - Bite force $200 \mathrm{~N}$

(c) Muscle deformation during molar biting

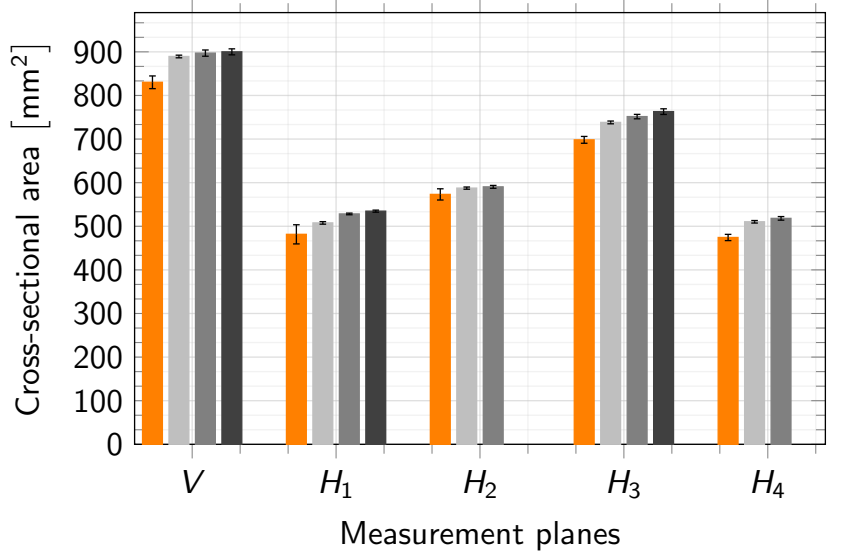

(d) Muscle deformation during incisor biting

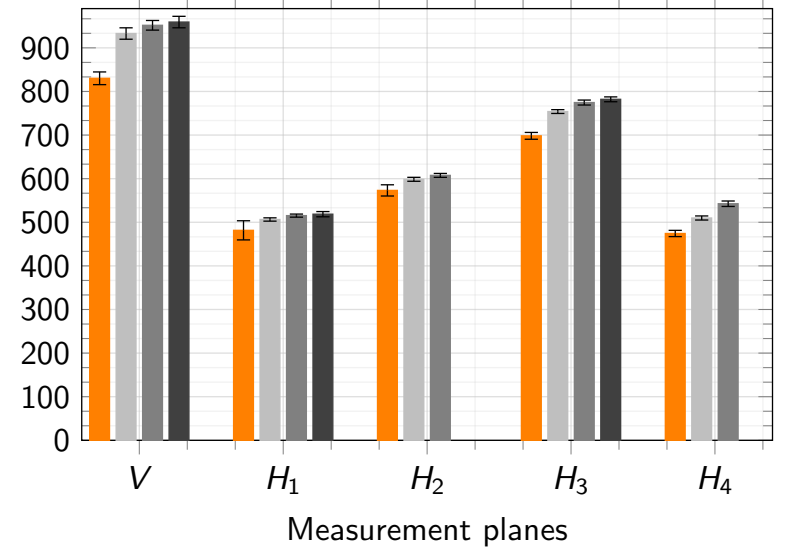

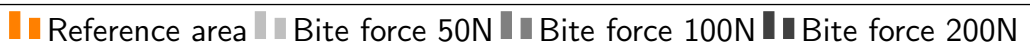

Fig. 4. (a/b) Masseter cross-sectional area change during molar and incisor biting in the horizontal plane $H_{3}$. The subject repeats at least two biting cycles per video sequence and is required to reach a bite force of $50 \mathrm{~N}, 100 \mathrm{~N}$, and $200 \mathrm{~N}$ in three individual video sequences. The optical flow algorithm tracks the manually segmented muscle contour through the entire image sequence. The inlet visualizes the procedure to determine relaxed and contracted cross-sectional muscle area. Through k-means clustering each curve is split into two groups, shown in orange and gray, for which the median is calculated, shown by dashed gray and orange lines, to obtain the data shown in (c/d). (c/d) Crosssectional areas in the five different measurement planes for molar and incisor biting. Bite force dependent evolution of area is compared to the respective reference area before muscle activation. K-means clustering is used to separate each curve into two subsets which are associated with the mean reference cross sectional area and the median cross sectional area at the respective bite force. For the bite force of $200 \mathrm{~N}$ no measurement data is available for horizontal cross sections $\mathrm{H}_{2}$ and $\mathrm{H}_{4}$ due to muscle fatigue, avoidance of sensor damage, and increased measurement uncertainty.

It should be noted here, that despite consistent experimental results the measurement setup is limited in ensuring the characterization of identical horizontal and vertical cross sections for every bite force level but much rather represents the characterization of material areas within a controlled measurement region. Secondly, the described muscle deformations were recorded for asymmetrical biting, thus associated with the placement of the force sensor between teeth on one side, without contralateral support.

\subsection{Numerical simulation of molar and incisor biting}

The numerical simulations are analyzed with respect to muscle shape changes for specific biting forces and biting positions. Bite forces are calculated from contact forces between those teeth that come into contact. In the human biting process the biting position is controlled by a superposed translation and rotation of the mandible about the temporomandibular joint. In the simulations presented here, biting is separated into two steps. In the first step, the mandible is translated along the sagittal plane in order to align the closing teeth. In the second step a rotation about the condyle head of the temporomandibular joint causes the mouth to close. 
(a) Molar biting

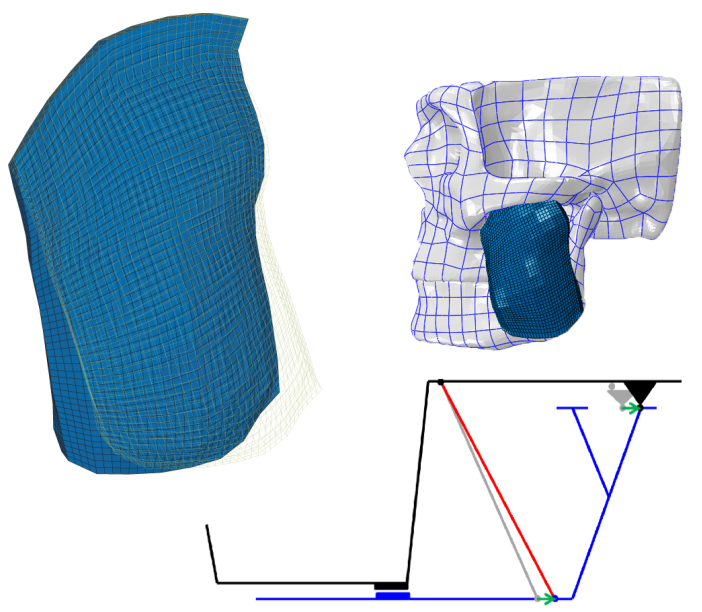

(b) Incisor biting

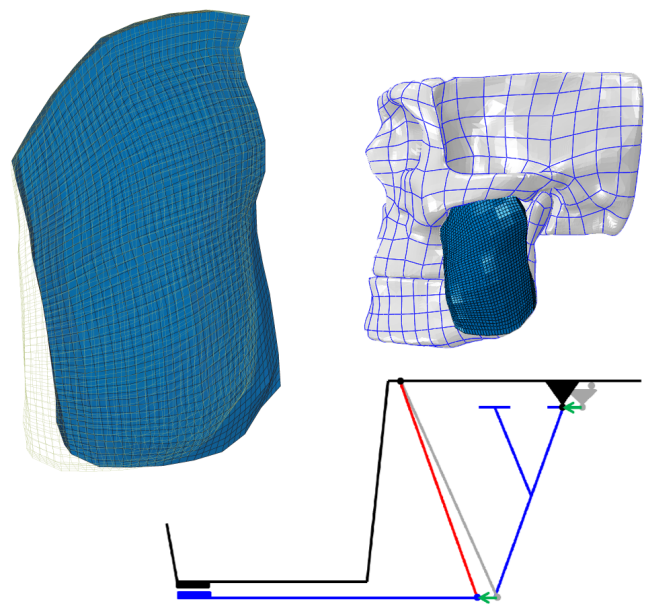

Fig. 5. Numerical simulation of biting including the differentiation between (a) molar and (b) incisor biting. The mechanical model described the jaw kinematics that depend on the biting location. In case of (a) molar biting the jaw must retract. In contrast, the jaw must protrude in the case of (b) incisor biting. This influences the initial shape of the masseter muscle before closing of the mouth as it is visualized by the resting state of the muscle (transparent green mesh) and the masseter shape at the beginning of biting (solid blue mesh).

The translation of the jaw is primarily driven by the pterygoid muscles during mandible protrusion and the temporalis muscles during mandible retraction, such that we assume the masseter muscle to deform passively during the first simulation step. Only in the second step the masseter is activated to initiate biting. The incorporation of other mastication muscles in the present model, together with a clear understanding of the muscle activation pattern, would allow to model biting in a single step. Fig. 5 shows two schematic representations of the numerical simulations: In the case of molar biting, the mandible is initially retracted, causing a passive stretch of the masseter muscle along its principal vertical direction; in the case of incisor biting, the mandible is protruded such that the frontal teeth touch when the jaw closes upon muscle activation.

Figure 6 shows the numerically predicted transient crosssectional area changes during the biting process for (a) molar and (b) incisor biting. It is observed that the vertical cross sections decrease similarly for increasing bite forces irrespective of the two biting positions. In the molar and incisor simulations all transverse muscle cross sections increase with bite force. The simulations show a rapid saturation of area change while bite forces continuously increase throughout the contraction step. The most caudal transverse plane $H_{4}$ shows lower effective area increase in comparison to cross sections $H_{1}-H_{3}$ and seems inhibited to thicken. At the same time, $\mathrm{H}_{2}$ and $\mathrm{H}_{3}$ experience the most pronounced thickening (up to 8\%) as this region of the muscle is least affected by boundary conditions. The kinematic constraints on the masseter muscle prohibit any muscle shortening once the teeth are in contact. In combination with the incompressibility condition of muscle tissue enforced at the integration point level, muscle deformations are limited to thickening in the middle and a thinning at the distal ends of the musclelatter not shown here. In fact, the numerical simulations pre- dict that the distal ends of the muscle become thinner with increasing bite force.

\section{Discussion}

This study combines experimental and numerical results for the analysis of mechanical masseter muscle response during biting. The initial average fiber stretch of the masseter muscle in the computational model is $\lambda_{m}=1.01[-]$ in the molar and $\lambda_{m}=0.994[-]$ in the incisor simulation. Similar values for fiber deformation were reported by Goto et al. [40] who experimentally observed a muscle length increase in case of pronounced jaw protrusion (i.e. noticeably beyond the necessary jaw protrusion for incisor biting) by a factor of 1.04. The constitutive muscle model presented in Section 2.2 incorporates the well-known relationship between active muscle fiber force and current fiber stretch. The output of force producing sarcomeres is dependent on the overlap of myosin and actin proteins [30]. Following the results of a previous study on masseter mechanics [29], maximum muscle motor force is obtained at a fiber stretch of $\lambda_{\text {opt }}=1.192[-]$. Based on this, the initial deformation of the masseter muscle in the incisor biting case is associated with an increased myosin-actin overlap and therefore lower maximum bite force. Conversely, molar biting is associated with an initial muscle stretch which allows for higher bite forces and reduced muscular deformations.

In general, experimental bite forces are obtained by the pressure sensor between the teeth, while numerically predicted bite force is calculated from contact forces between those teeth that come into contact. Muscle force is calculated as the sum of all reaction forces exerted on the cranial anchoring of the massemuter muscle during contraction, see Fig. 2c. The mechanical load on the condyle head is given by the reaction forces and moments or a simple balance of 
(a) Simulated area changes for molar biting

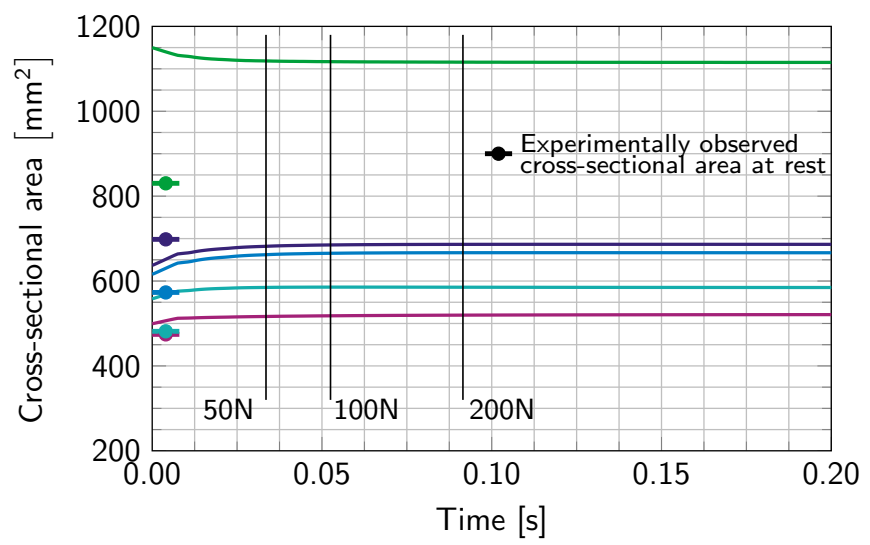

(b) Simulated area changes for incisor biting

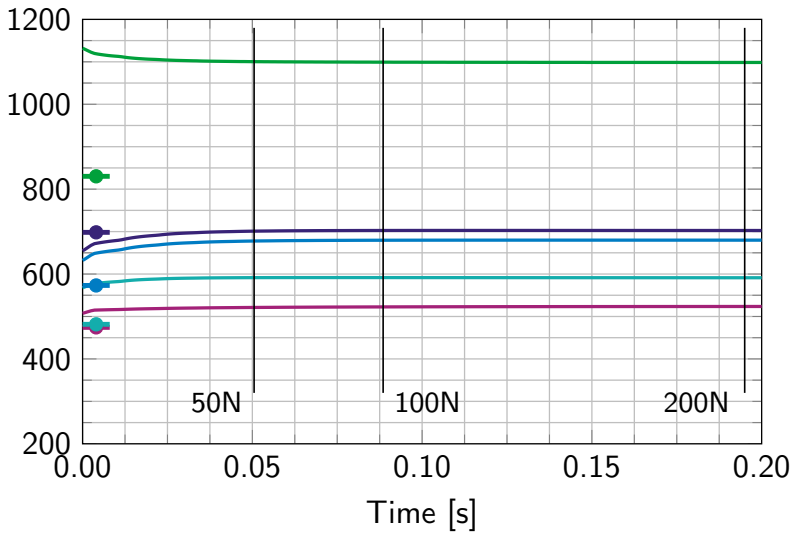

$-V-H_{1}-H_{2}-H_{3}-H_{4}$

(c) Average of $H_{2}$ and $H_{3}$

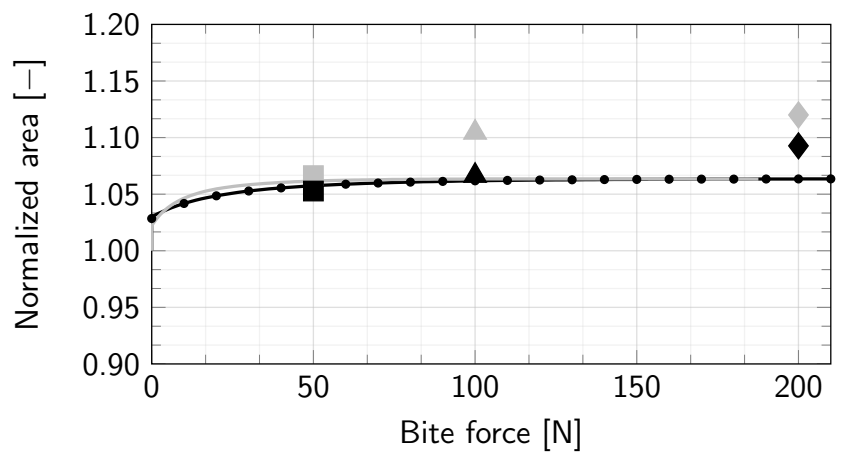

(d) Measurement plane: $V$

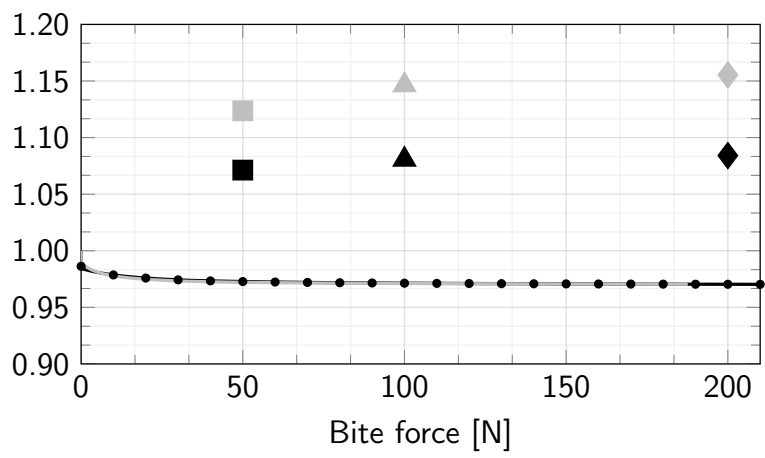

$$
\begin{array}{lll}
\rightarrow \text { Molar simulations } & \mathbf{Q} \Delta \diamond & \text { Molar measurements } \\
\longrightarrow \text { Incisor simulations } & \square \Delta \diamond & \text { Incisor measurements }
\end{array}
$$

Fig. 6. (a/b) Numerically predicted muscle deformation in terms of area change for increasing bite force. There is noticeable variation in the simulated shape change depending on biting position and measurement location within the masseter muscle. Cross-sectional area is approximated by the convex hull encasing the nodes of the masseter surface intersecting with the respective measurement planes. The experimentally observed cross-sectional area of the masseter in the resting state $(t=0$, reference area) is shown. (c/d) Comparison of numerically predicted and experimentally observed muscle deformation with respect to bite force. Deformation shown in terms of normalized cross-sectional area of (a) the average of $\mathrm{H}_{2}$ and $\mathrm{H}_{3}$ and (b) the vertical measurement plane $\mathrm{V}$.

forces, respectively. The activation time to reach set bite forces depends on the material parameters of the contraction velocity function $f_{v}$, the local density of muscle fibers $n_{M U}$, amongst others (Eq. 6). All parameters were set according to Weickenmeier et al. [29].

Tab. 1 shows the maximum bite forces which demonstrate good agreement between experimental data and the corresponding numerical models. The difference between maximum molar $(363 \mathrm{~N})$ and incisor $(211 \mathrm{~N})$ bite force is explained by mastication kinematics: the force equilibrium within the mastication system depends on the lever arm of the muscle force with respect to the condyle head and bite position. The lever arm of the bite force vector is significantly smaller during molar biting leading to a noticeably more effective conversion of muscle force into bite forcean inherent advantage during chewing and grinding of food between molars. Thus, equal muscle force leads to lower bite force for incisor as compared to molar biting, due to the moment equilibrium equation $[15,16]$.

Literature reports on numerous experimental measurements of maximum bite forces [41-43]. The range of reported data $(>500 \mathrm{~N})$ is related to different force sensors, measurement positions, and subject demographics. In the present study, the experimentally measured maximum bite force of $363 \mathrm{~N}$ in the molar and $211 \mathrm{~N}$ in the incisor biting case match values by Palinkas et al. [41] of $343.5 \mathrm{~N}$ for molar biting and by Paphangkorakit et al. [42] of $210 \mathrm{~N}$ for incisor biting remarkably well. In particular, Paphangkorakit et al. [42] use a force sensor very similar to the present device with respect to sensor thickness and stabilization of the desired bite position during rapid force generation.

Few physics-based computational models exist that investigate the mechanical masseter response during mastication [4, 28, 44]. Röhrle et al. [28] predict maximum 


\begin{tabular}{|l|l|c|}
\hline location & mode & maximum bite force \\
\hline \hline molar & experiment & $363 \mathrm{~N}$ \\
molar & simulation & $366 \mathrm{~N}$ \\
\hline \hline incisor & experiment & $211 \mathrm{~N}$ \\
incisor & simulation & $187 \mathrm{~N}$ \\
\hline
\end{tabular}

Table 1. Experimentally observed and numerically predicted maximum molar and incisor bite forces.

masseter forces that are significantly lower $(77 \mathrm{~N})$ in comparison to the present study. The authors suggest, however, that their results- being at the lower bound of bite forces reported in literature- may be associated with their choice of model parameters as well as the comparison of biting data with clenching experiments. In fact, the initial shear modulus considered by Röhrle et al. [28] differs from the value used in this study by one order of magnitude. The agreement between numerical and experimental maximum bite force is remarkable, especially since no parameter fitting was performed. This indicates that the proposed model provides a reliable representation of the clenching process.

The two different initial masseter muscle positions for incisor and molar biting have a noticeable impact on the kinematic configuration at the beginning of the active biting step, as shown in Fig. 5. In particular, horizontal crosssectional areas in the reference configuration, i.e. prior to activation, are significantly lower compared to the biting phase, as shown in Fig. 6a/b. In terms of absolute reference area the experimental and numerical cross-sectional area differ by $18 \%$ for $H_{1}, 7 \%$ for $H_{2}, 10 \%$ for $H_{3}, 6 \%$ for $H_{4}$, and $36 \%$ for the vertical plane $V$. These differences are associated with a mismatch of the experimental measurement plane and the cross section analyzed in the numerical simulation. Additionally, they are associated with differences in the visualization of soft anatomical structures in the two imaging modalities used in this study. It is well understood that ultrasound imaging potentially under-represents the muscletendon complex thus leading to an underestimation of the vertical cross-sectional area. A normalization of the crosssectional areas with respect to reference areas allows for a comparison of transient area changes at the three specific bite forces. In general, the masseter muscle requires significantly more activation energy to obtain the incisor bite force of $200 \mathrm{~N}$ in comparison to molar biting. Correspondingly, experimentally observed horizontal cross-sectional areas are found to increase on average by a maximum of $8 \%$ for molar biting and $12 \%$ for incisor biting. The calculation predicts $7 \%$ increase for both molar and incisor biting. While the model provides a three-dimensional representation of muscle deformations during contraction, it cannot fully explain the observed cross-sectional area changes. The model treats the masseter muscle as an incompressible tissue, such that horizontal cross-sectional area increase coincide with an area decrease in transverse planes. This additional model limitation explains the results reported in Fig. 6c/d; the predicted vertical cross-sectional area decrease contradicts the exper- imentally observed area increase. More detailed anatomical information would allow to improve model predictions, in particular concerning the representation of the boundary conditions reflecting the insertion points, the muscle-tendon complex (currently considered as a pure muscle structure), as well as the local fiber orientation within the muscle.

The present study is further limited by considering a single subject. While it was the primary focus to assess the feasibility of a combined experimental and computational approach to understanding masseter muscle behavior, future work must contain a multi-subject comparison to verify conclusions drawn from this study. Future volunteers should also be trained to reproduce a physiological biting motion during the muscle deformation and bite force measurement, in order to minimize the impact of increased motor cortex control of mastication muscle activation. The current experimental setup, prescribing a target bite force and using visual feedback to inform the subject on their bite force, leads to a potential cortical over-regulation of muscle activation and will therefore have influenced the observed muscle response in the present study.

\section{Conclusion}

The present study includes a comprehensive experimental and numerical investigation of the mechanical behavior of the masseter muscle. An anatomically detailed finite element model of the mastication system was created; semiautomatic segmentation of medical images, subsequent mesh generation, and the definition of physically motivated kinematic constraints constitute the individual steps in the preparation of a realistic geometric representation of the masseter muscle, mandible, and skull. Incorporating the numerical implementation of a phenomenological constitutive model for skeletal muscle tissue provided a tool for reliable prediction of active muscle response in mastication and jaw kinematics. Boundary conditions and kinematic constraints were imposed on the muscle and the temporomandibular joint in accordance with anatomical features presented in literature. An experimental setup was developed that allows for simultaneous bite force measurements and ultrasound-based visualization of masseter muscle deformations during biting. Four horizontal and one vertical measurement plane were defined in order to approximate the three-dimensional muscle response during molar and incisor biting. Muscle crosssectional area change was observed to depend on biting location and bite force magnitude. Similarly, numerically predicted bite forces and muscle deformations were found to be bite-position dependent as well. In terms of absolute values, experiments and simulation showed noticeable differences in the cross-sectional area in the reference state, in particular for the vertical plane $V$. This is attributed to differences in the visualization of soft anatomical structures such as the muscle tendon complex in the two imaging modalities used in this study. After normalization with respect to the reference area, a similar cross-sectional area increase of horizontal planes $\mathrm{H}_{2}$ and $\mathrm{H}_{3}$ is observed in both experiments and simulations. The numerically predicted maximum bite forces (molar: 
$366 \mathrm{~N}$, incisor: $187 \mathrm{~N}$ ) showed remarkable agreement with the experimentally observed values (molar: $363 \mathrm{~N}$, incisor: $211 \mathrm{~N}$ ) although no parameter optimization was performed. As a next step, accounting for the contribution of the temporalis muscle would enhance force and deformation predictions by the finite element model [5]. Further improvements could be obtained through a systematic quantification of muscle recruitment patterns during mastication in dependence of biting location and bite force using electromyography $[11,45]$. Electromyography measurements could be useful to determine the changes in muscle activation patterns associated with eating and temporomandibular joint disorders [2, 46, 47]. Moreover, muscle shape changes including shortening and cross-sectional thickening during contraction have been shown to correlate with EMG data in experiments with minipigs [48]. The present ultrasound data should be evaluated in combination with EMG data on the same subject using the setup presented by Le Révérend et al. [11] to confirm those findings. Such EMG measurements could also help calibrate the contributions of the masseter and temporalis muscle to inform more complex biting simulations. Moreover, determination of the location specific muscle fiber orientation of masseter and temporalis muscle through diffusion tensor imaging would allow to adequately incorporate muscle fiber anisotropy in the numerical simulations.

\section{References}

[1] Williams, P., et al., 1995. Gray's Anatomy: The Anatomical Basis of Medicine and Surgery. Churchill Livingstone New York.

[2] Chaves, T. C., dos Santos Aguiar, A., Felicio, L. R., Greghi, S. M., Regalo, S. C. H., and Bevilaqua-Grossi, D., 2017. "Electromyographic ratio of masseter and anterior temporalis muscles in children with and without temporomandibular disorders". International Journal of Pediatric Otorhinolaryngology, 97, pp. 35-41.

[3] Cheng, H.-Y., Peng, P.-W., Lin, Y.-J., Chang, S.-T., Pan, Y.-N., Lee, S.-C., Ou, K.-L., and Hsu, W.-C., 2013. "Stress analysis during jaw movement based on vivo computed tomography images from patients with temporomandibular disorders". International Journal of Oral and Maxillofacial Surgery, 42(3), pp. 386-392.

[4] Commisso, M., Martínez-Reina, J., Ojeda, J., and Mayo, J., 2015. "Finite element analysis of the human mastication cycle". Journal of the Mechanical Behavior of Biomedical Materials, 41, pp. 23-35.

[5] Ackland, D. C., Robinson, D., Redhead, M., Lee, P. V. S., Moskaljuk, A., and Dimitroulis, G., 2017. "A personalized 3d-printed prosthetic joint replacement for the human temporomandibular joint: From implant design to implantation". Journal of the Mechanical Behavior of Biomedical Materials, 69, pp. 404-411.

[6] Narra, N., Valášek, J., Hannula, M., Marcián, P., Sándor, G., Hyttinen, J., and Wolff, J., 2014. "Finite element analysis of customized reconstruction plates for mandibular continuity defect therapy". Journal of Biomechanics, 47(1), pp. 264-268.
[7] Martinez Choy, S., Lenz, J., Schweizerhof, K., Schmitter, M., and Schindler, H., 2017. "Realistic kinetic loading of the jaw system during single chewing cycles: a finite element study". Journal of Oral Rehabilitation, 44(5), pp. 375-384.

[8] Hirose, M., Tanaka, E., Tanaka, M., Fujita, R., Kuroda, Y., Yamano, E., Van Eijden, T., and Tanne, K., 2006. "Three-dimensional finite-element model of the human temporomandibular joint disc during prolonged clenching”. European Journal of Oral Sciences, 114(5), pp. 441-448.

[9] Mowlavi, S., Engmann, J., Burbidge, A., Lloyd, R., Hayoun, P., Le Reverend, B., and Ramaioli, M., 2016. "In vivo observations and in vitro experiments on the oral phase of swallowing of newtonian and shearthinning liquids". Journal of Biomechanics, 49(16), pp. 3788-3795.

[10] Hayoun, P., Engmann, J., Mowlavi, S., Le Révérend, B., Burbidge, A., and Ramaioli, M., 2015. "A model experiment to understand the oral phase of swallowing of newtonian liquids". Journal of Biomechanics, 48(14), pp. 3922-3928.

[11] Le Révérend, B., Saucy, F., Moser, M., and Loret, C., 2016. "Adaptation of mastication mechanics and eating behaviour to small differences in food texture". Physiology \& behavior, 165, pp. 136-145.

[12] Osborn, J., and Baragar, F., 1985. "Predicted pattern of human muscle activity during clenching derived from a computer assisted model: Symmetric vertical bite forces". Journal of Biomechanics, 18(8), pp. 599-612.

[13] Koolstra, J., and Van Eijden, T., 2001. "A method to predict muscle control in the kinematically and mechanically indeterminate human masticatory system". Journal of Biomechanics, 34(9), pp. 1179-88.

[14] Hannam, A., Stavness, I., Lloyd, J., and Fels, S., 2008. "A dynamic model of jaw and hyoid biomechanics during chewing". Journal of Biomechanics, 41(5), pp. 1069-1076.

[15] Osborn, J., 1996. "Features of human jaw design which maximize the bite force". Journal of Biomechanics, 29(5), pp. 589-595.

[16] Le Révérend, B., and Hartmann, C., 2014. "Numerical modeling of human mastication, a simplistic view to design foods adapted to mastication abilities". Physiology and Behavior, 124, pp. 61-64.

[17] Hill, A., 1938. "The heat of shortening and the dynamic constants of muscle". Proceedings of the Royal Society of London. Series B, Biological Sciences, 126, pp. 136195.

[18] Van Eijden, T., and Raadsheer, M., 1992. "Heterogeneity of fiber and sarcomere length in the human masseter muscle". The Anatomical Record, 232(1), pp. 78-84.

[19] Van Eijden, T., Korfage, J., and Brugman, P., 1997. "Architecture of the human jaw-closing and jawopening muscles". The Anatomical Record, 248(3), pp. 464-474.

[20] Gröning, F., Jones, M. E., Curtis, N., Herrel, A., O’Higgins, P., Evans, S. E., and Fagan, M. J., 2013. 
"The importance of accurate muscle modelling for biomechanical analyses: a case study with a lizard skull”. Journal of The Royal Society Interface, 10(84), p. 20130216.

[21] Böl, M., Ehret, A. E., Leichsenring, K., Weichert, C., and Kruse, R., 2014. "On the anisotropy of skeletal muscle tissue under compression”. Acta Biomaterialia, 10(7), pp. 3225-3234.

[22] Hodgson, J. A., Chi, S.-W., Yang, J. P., Chen, J.-S., Edgerton, V. R., and Sinha, S., 2012. "Finite element modeling of passive material influence on the deformation and force output of skeletal muscle". Journal of the Mechanical Behavior of Biomedical Materials, 9, pp. 163-183.

[23] Commisso, M., Calvo-Gallego, J., Mayo, J., Tanaka, E., and Martínez-Reina, J., 2016. "Quasi-linear viscoelastic model of the articular disc of the temporomandibular joint". Experimental Mechanics, 56(7), pp. 1169-1177.

[24] Iwasaki, L., Gonzalez, Y., Liu, Y., Liu, H., Markova, M., Gallo, L., and Nickel, J., 2017. "Tmj energy densities in healthy men and women". Osteoarthritis and Cartilage.

[25] Lamela, M. J., Pelayo, F., Ramos, A., FernándezCanteli, A., and Tanaka, E., 2013. "Dynamic compressive properties of articular cartilages in the porcine temporomandibular joint". Journal of the Mechanical Behavior of Biomedical Materials, 23, pp. 62-70.

[26] Berranen, Y., Hayashibe, M., Guiraud, D., and Gilles, B., 2014. "Real-time muscle deformation via decoupled modeling of solid and muscle fiber mechanics". pp. 65-72.

[27] Böl, M., Leichsenring, K., Weichert, C., Sturmat, M., Schenk, P., Blickhan, R., and Siebert, T., 2013. "Threedimensional surface geometries of the rabbit soleus muscle during contraction: Input for biomechanical modelling and its validation". Biomechanics and Modeling in Mechanobiology, 12(6), pp. 1205-1220.

[28] Röhrle, O., and Pullan, A., 2007. "Three-dimensional finite element modelling of muscle forces during mastication". Journal of Biomechanics, 40(15), pp. 33633372.

[29] Weickenmeier, J., Itskov, M., Mazza, E., and Jabareen, M., 2014. "A physically motivated constitutive model for 3D numerical simulation of skeletal muscles". International Journal for Numerical Methods in Biomedical Engineering, 30(5), pp. 545-562.

[30] Ehret, A. E., Böl, M., and Itskov, M., 2011. “A continuum constitutive model for the active behaviour of skeletal muscle". Journal of the Mechanics and Physics of Solids, 59(3), pp. 625-636.

[31] Barbarino, G., Jabareen, M., Trzewik, J., Nkengne, A., Stamatas, G., and Mazza, E., 2009. "Development and validation of a three-dimensional finite element model of the face". Journal of biomechanical engineering, 131(4), p. 041006.

[32] ABAQUS, 2009. Abaqus/Standard Analysis User's Manual. Dassault Systmes, Providence, RI, USA.
[33] Mao, J., and Osborn, J., 1994. "Direction of a bite force determines the pattern of activity in jaw-closing muscles". Journal of Dental Research, 73(5), pp. 11121120.

[34] Kubo, K., Kawata, T., Ogawa, T., Watanabe, M., and Sasaki, K., 2006. "Outer shape changes of human masseter with contraction by ultrasound morphometry". Archives of Oral Biology, 51(2), pp. 146-153.

[35] McMillan, A., and Hannam, A., 1992. "Task-related behaviour of motor units in different regions of the human masseter muscle". Archives of Oral Biology, 37(10), pp. 849-857.

[36] Corless, R., Gonnet, G., Hare, D., Jeffrey, D., and Knuth, D., 1996. "On the Lambert $W$ function". Advances in Computational Mathematics, 5(4), pp. 329359.

[37] Weickenmeier, J., Wu, R., Lecomte-Grosbras, P., Witz, J.-F., Brieu, M., Winklhofer, S., Andreisek, G., and Mazza, E., 2014. "Experimental characterization and simulation of layer interaction in facial soft tissues". Lecture Notes in Computer Science, 8789, pp. 233-241.

[38] Serra, M., Duarte Gaviao, M., and dos Santos Uchoa, M., 2008. "The use of ultrasound in the investigation of the muscles of mastication". Ultrasound in Medicine \& Biology, 34(12), pp. 1875-1884.

[39] Raadsheer, M., Van Eijden, T., Van Spronsen, P., Van Ginkel, F., Kiliaridis, S., and Prahl-Andersen, B., 1994. "A comparison of human masseter muscle thickness measured by ultrasonography and magnetic resonance imaging". Archives of Oral Biology, 39(12), pp. 1079-1084.

[40] Goto, T., Langenbach, G., and Hannam, A., 2001. "Length changes in the human masseter muscle after jaw movement". The Anatomical Record, 262(3), pp. 293-300.

[41] Palinkas, M., Nassar, M., Cecílio, F., Siéssere, S., Semprini, M., Machado-de Sousa, J., Hallak, J., and Regalo, S., 2010. "Age and gender influence on maximal bite force and masticatory muscles thickness". Archives of Oral Biology, 55(10), pp. 797-802.

[42] Paphangkorakit, J., and Osborn, J., 1997. "The effect of pressure on a maximum incisal bite force in man". Archives of Oral Biology, 42(1), pp. 11-17.

[43] Varga, S., Spalj, S., Varga, M., Milosevic, S., Mestrovic, S., and Slaj, M., 2011. "Maximum voluntary molar bite force in subjects with normal occlusion". The European Journal of Orthodontics, 33(4), pp. 427-433.

[44] Marková, M., and Gallo, L. M., 2016. "The influence of the human TMJ eminence inclination on predicted masticatory muscle forces". Human Movement Science, 49, pp. 132-140.

[45] van Dijk, J., Eiglsperger, U., Hellmann, D., Giannakopoulos, N., McGill, K., Schindler, H., and Lapatki, B., 2016. "Motor unit activity within the depth of the masseter characterized by an adapted scanning emg technique". Clinical Neurophysiology, 127(9), pp. 3198-3204.

[46] Politti, F., Casellato, C., Kalytczak, M. M., Garcia, M. 
B. S., and Biasotto-Gonzalez, D. A., 2016. "Characteristics of emg frequency bands in temporomandibullar disorders patients". Journal of Electromyography and Kinesiology, 31, pp. 119-125.

[47] Shimada, A., Baad-Hansen, L., and Svensson, P., 2015. "Effect of experimental jaw muscle pain on dynamic bite force during mastication". Archives of oral biology, 60(2), pp. 256-266.

[48] Dutra, E. H., Caria, P. H., Rafferty, K. L., and Herring, S. W., 2010. "The buccinator during mastication: a functional and anatomical evaluation in minipigs". Archives of oral biology, 55(9), pp. 627-638. 